\title{
Carnets
}

Revue électronique d'études françaises de l'APEF

Deuxième série - 9 | 2017

Reconnaissances et légitimité en français

\section{Littérature et sciences cognitives : apports et légitimité d'une lecture transversale}

\section{Gabriella Bandura}

\section{(2) OpenEdition}

Journals

\section{Édition électronique}

URL : http://journals.openedition.org/carnets/2113

DOI : 10.4000/carnets. 2113

ISSN : 1646-7698

Éditeur

APEF

\section{Référence électronique}

Gabriella Bandura, «Littérature et sciences cognitives : apports et légitimité d'une lecture transversale », Carnets [En ligne], Deuxième série - 9 | 2017, mis en ligne le 31 janvier 2017, consulté le 19 avril 2019. URL : http://journals.openedition.org/carnets/2113; DOI : 10.4000/carnets.2113

Ce document a été généré automatiquement le 19 avril 2019.

\section{(c) (i) (8)}

Carnets est mis à disposition selon les termes de la licence Creative Commons - Atribution - Pas d'utilisation commerciale 4.0 International. 


\title{
Littérature et sciences cognitives : apports et légitimité d'une lecture transversale
}

\author{
Gabriella Bandura
}

\section{Vers une approche cognitive}

Depuis quelques années, il y a une propagation de diverses approches du texte littéraire à travers d'autres disciplines relevant des sciences humaines et des sciences dures également, et ces rapprochements jouissent de la reconnaissance de l'appareil légitimateur. Les sciences cognitives, qui ont pour objet l'étude scientifique du fonctionnement de la connaissance, autrement dit de la cognition, font partie de ces disciplines.

2 Selon le neurobiologiste Francisco Varela, expert mondialement reconnu dans le domaine, il s'agit d'une entreprise technologique et conceptuelle considérable qui regroupe elle-même une multitude de disciplines «inter-résonnantes » (Varela, 1989a: 21), notamment la linguistique, les neurosciences, la philosophie de l'esprit, l'intelligence artificielle, etc., qui tentent de modéliser la connaissance humaine selon leurs propres préoccupations et engagements. En même temps, les modèles qu'elles produisent sont inséparables des avancées technologiques contemporaines transformant les pratiques socioculturelles qui les sous-tendent. Le développement de l'intelligence artificielle en est l'exemple le plus visible.

3 Mais l'impact des sciences cognitives se propage dans le champ de la littérature également, où depuis les années quatre-vingt, les études du texte à travers l'appareillage conceptuel de la cognition ne cessent de se multiplier. Ce qui légitime cette ouverture, ce sont les possibilités d'élargissement des techniques de lecture que les sciences cognitives proposent, en nous incitant « à prendre en compte de façon beaucoup plus minutieuse la réalité de l'acte de lecture » (Abrioux, $1990: 6$ ). 
4 L'évolution des modèles de cette entreprise conceptuelle récente reflète le fonctionnement dynamique et contingent de la cognition humaine, et dans un sens plus large les orientations et les sensibilités de la pensée contemporaine, caractérisée, elle aussi, par cette perspective dynamique. Ceci est surtout vrai pour la littérature française contemporaine et de l'extrême contemporain, basée sur l'hétérogénéité et une « ontologie de l'incertitude radicale » (Westphal, $2007: 12$ ).

5 Nous allons réinterroger ici l'apport et la légitimité de cette interférence entre la littérature et les sciences cognitives, en effectuant d'abord une lecture cognitive d'un roman français contemporain, La Possibilité d'une île de Michel Houellebecq, pour souligner ensuite, ce que cette même littérature peut apporter, à son tour, à l'étude de la cognition. Notre lecture s'appuiera sur le modèle autopoiétique, élaboré par les neurobiologistes Francisco Varela et Humberto Maturana ${ }^{1}$.

\section{Qu'est-ce que l'autopoïèse?}

6 Le modèle de l'autopoïèse constitue le fondement biologique du troisième grand paradigme des sciences cognitives, appelé enaction ${ }^{2}$, qui diffère des deux autres paradigmes plus statiques ${ }^{3}$, puisqu'il conçoit la cognition non plus comme une représentation du monde extérieur préexistant, mais comme la co-émergence de l'esprit incarné (c'est-à-dire de l'humain) et du monde, dans leurs interactions diverses. Ceci est possible grâce au comportement de cet esprit incarné comme une unité autopoiétique, autrement dit à sa capacité à l'autoproduction dans ses échanges avec le monde extérieur.

7 Il s'agit donc d'un modèle qui formalise le fonctionnement cognitif du vivant (allant des unicellulaires jusqu'à l'individu) comme un processus d'autoproduction ${ }^{4}$ : «(...) une machine autopoiétique engendre et spécifie continuellement sa propre organisation. Elle accomplit ce processus incessant de remplacement de ses composants, parce qu'elle est continuellement soumise à des perturbations externes (je souligne), et constamment forcée de compenser ces perturbations » (Varela 1989b : 45).

8 En réponse à celles-ci, le vivant produit une clôture opérationnelle, autrement dit une frontière dynamique qui lui permet de réagir aux perturbations et de les intégrer dans son système, c'est-à-dire de se transformer continuellement tout en restant le même : une entité qui se distingue du monde environnant. Ainsi le système autopoiétique satisfait un critère paradoxal: il maintient la concomitance entre son organisation, c'est-à-dire l'ensemble des éléments invariants qui constituent sa configuration globale, et sa structure, l'univers indéfini des variables à l'origine des changements permanents :

Un système autopoiétique est organisé comme un réseau de processus de production de composants qui (a) régénèrent continuellement par leurs transformations et leurs interactions le réseau qui les a produits, et qui (b) constituent le système en tant qu'unité concrète dans l'espace où il existe, en spécifiant le domaine topologique où il se réalise comme réseau (ibid.).

9 La clôture opérationnelle est un proche parent du feed-back des systèmes cybernétiques à entrées et sorties, mais cependant elle s'en diffère, puisqu'elle n'a pas besoin d'une source de référence externe : le système produit lui-même les informations qui le constituent, en fonction des perturbations qui le touchent. Autrement dit, la clôture est cette frontière par laquelle le système se définit comme une entité autonome. 
Cette autonomie basée sur un mécanisme de régulation interne permet de dépasser le cadre de l'homéostasie ${ }^{5}$ qui est au cœur du développement cybernétique :

Les théories classiques de l'auto-régulation ou de l'homéostasie reposaient sur la définition d'un état stable contre des perturbations susceptibles de l'en écarter. Au contraire, ici, il s'agit de comprendre non seulement comment le système fait pour se maintenir dans un état stable, mais comment il fait pour se transformer luimême (Atlan, Castoriadis, Domenach et al., 1987 : 19).

11 Le modèle autopoiétique de Varela et Maturana décrit ainsi le fonctionnement du vivant comme une dynamique d'individuation, autrement dit de singularisation, qui transcende l'opposition entre stabilité et changement, et évolue de manière métastable ${ }^{6}$. L'apport de son transfert dans le champ littéraire réside justement dans son aspect métastable apte à capturer les postures d'individuation à l'œuvre dans les textes.

\section{Une lecture cognitive : l'île de Houellebecq}

12 Michel Houellebecq, l'auteur de La Possibilité d'une île (Fayard, 2005), est un des écrivains contemporains les plus connus et traduits dans le monde, mais aussi «l'auteur le plus controversé du paysage littéraire français actuel » (Wesemael, Clément, 2007 : quatrième de couverture). Ses romans suscitent la polémique et de violentes critiques à cause de ses positions idéologiques volontiers provocatrices, mais en même temps sa reconnaissance par l'appareil légitimateur est indubitable. Il fait partie des programmes universitaires, il est le sujet de nombreux mémoires et thèses et il est le détenteur de plusieurs prix littéraires, dont le Goncourt pour La carte et le territoire, en 2010.

Dans La Possibilité d'une île, comme dans ses livres précédents, Houellebecq ausculte les névroses et les pathologies de la société occidentale. À travers la vie de son protagoniste Daniel 1, il esquisse « l'extension du domaine de la lutte » de toute une génération coincée dans la machine de la compétitivité néo-capitaliste, qui a broyé à une vitesse fulgurante toutes les valeurs ayant trait à l'humain et à sa capacité à aimer. Mais ce qui est intéressant ici, est qu'un nouveau système complexe et mystérieux est en train de surgir, qui n'a pas pour objectif final de se focaliser sur la perspective du bonheur manqué et de proposer une possibilité de solution pour y remédier.

Ce nouveau système s'élabore par la création d'espaces alternatifs science-fictionnels, qui s'intègrent continuellement dans l'espace référentiel, le tout ressemblant à un système vivant, dynamique qui s'auto-crée sans cesse. Toutefois, il ne s'agit pas, dans notre lecture, d'un système déjà configuré qui devient dynamique par la transgression du cadre réaliste, mais plutôt de relations de concaténation continuelle, de rencontres entre espaces alternatifs de la fiction qui font émerger tout un nouveau régime dont les agencements ne cessent de s'individuer, sous le signe de potentiels imprévisibles, à venir.

Ces agencements d'individuation se forment progressivement aux lieux de croisements de plusieurs « récits de vie » : le récit référentiel de Daniel 1 qui vit dans le monde actuel et les récits science-fictionnels de ses prolongements néo-humains Daniel 24 et Daniel 25. Ces deux derniers, conçus à partir du génome de Daniel 1 par l'équipe de la secte élohimite ${ }^{8}$, vivent deux millénaires plus tard sur la planète ravagée par une apocalypse nucléaire et leur tâche principale est de lire et de commenter les écrits de leurs prédécesseurs. Chaque être de la lignée de clones des Daniel est fabriqué à partir de l'ADN de son prédécesseur s'étant suicidé, qu'il remplace en se retrouvant dans un corps jeune et plein de vitalité. 
16 La génétique permet donc de cristalliser la perspective de la vie éternelle dans le roman, mais ce qui nous intéresse particulièrement est le système d'entrelacement des récits qui suscite l'émergence d'un régime complexe, non-standard. Le récit de vie, « au fond assez proche de ce qu'on appelait jadis l'autobiographie» (PI, 27), sert essentiellement à maintenir la continuité mémorielle et donc identitaire entre les êtres de chaque lignée de clones en attendant l'élaboration du transfert moléculaire direct, mais simultanément il constitue « un seuil »" (Westphal, $2007: 163$ ) qui donne accès à une circulation libre et des connexions imprédictibles entre l'espace référentiel et celui de la science-fiction.

17 Ainsi les récits de vie néo-humains qui ne cessent de se proliférer dans le texte et de coexister, voire fusionner avec le récit de Daniel 1, peuvent être conçus comme une suite de perturbations qui contribuent à élaborer, à tisser ce système complexe. Les perturbations ne sont pas considérées ici comme des facteurs qui dérangent, mais comme des éléments que le système « accueille » et qui prennent part dans la constitution et les transformations de ce dernier. Autrement dit, ces récits de science-fiction, par leurs activités de perturbations, contribuent à l'élaboration d'une clôture opérationnelle, d'un ordre à partir de l'instabilité.

Les récits de vie des néo-humains apparaissent et se tissent effectivement dans un rapport de contiguïté avec l'espace référentiel dès le début du roman, en suscitant continuellement le fonctionnement opérationnel et, partant, «l'explication opérationnelle $»^{10}$ (Kremer-Marietti, 2012:5) de ce dernier. Cela veut dire qu'il y a une élaboration d'une clôture qui n'apparait pas comme une frontière définitive, mais comme une clôture opérationnelle dynamique, une réponse toujours retardée en fonction des connexions et des agencements imprévisibles, à venir, qui se forment dans l'entre-deux de ces espaces alternatifs.

Le monde complexe de La Possibilité qui est en train d'émerger évoque ainsi le fonctionnement du domaine cognitif du vivant, celui des systèmes autopoiétiques, puisqu'il se construit et se spécifie en tant que système, en tant que clôture, dans un processus de régénération permanente, par l'ouverture à l'imprévisible, à l'instable. L'autoproduction de ce monde peut être illustrée tout simplement par des cercles où les espaces du récit de vie réaliste de Daniel 1 et les récits science-fictionnels évoluent dans des rapports de contiguïté, de fusion (cf. figure a) : 


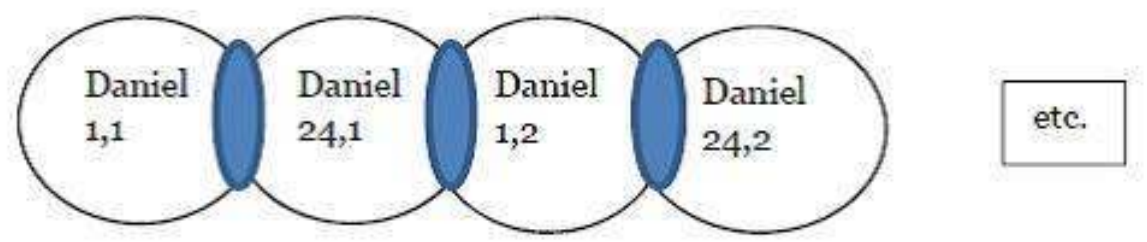

DANIEL 1,1: Comme ils restent présents à ma mémoire, les premiers instants de ma vocation de bouffon! J'avais alors dix-sept ans, et je passais un mois d'août plutôt morne dans un club all inclusive en Turquie (...) J'avais commencé par des petits sketches sur les familles recomposées, les journalistes du Monde (...) $(P I, 19,21)$.

DANIEL 24,1: Regarde les petits êtres qui bougent dans le lointain ; regarde. Ce sont des hommes. Dans la lumière qui décline, j'assiste sans regret à la disparition de l'espèce. Un dernier rayon de soleil rase la plaine, passe au-dessus de la chaîne montagneuse qui barre l'horizon vers l'Est, teinte le paysage désertique d'un halo rouge. Les treillages métalliques de la barrière de protection qui entoure la résidence étincellent $(P I, 26)$.

DANIEL 1,2: II m'est à peu près impossible aujourd'hui de me souvenir pourquoi j'ai épousé ma première femme; si je la croisais dans la rue, je ne pense même pas que je parviendrais à la reconnaître. On oublie certaines choses, on les oublie réellement ; $(P l, 28)$.

DANIEL 24,2 : Aujourd'hui que tout apparaît dans la clarté du vide, j'ai la liberté de regarder la neige. C'est mon lointain prédécesseur, l'infortuné comique, qui avait choisi de vivre ici $(. .).(P I, 42)$.

Dans leurs interconnexions, tout un nouveau système est en train de jaillir et de se (re)configurer, dont les espaces se façonnent d'une manière récursive, mais ne restent jamais les mêmes, en étant craquelés par des potentiels chaotiques, fluctuants, qu'on pourrait concevoir comme des poches métastables (cf. les parties bleues sur la figure).

Ces poches évoluent dans le sillon, dans la concomitance du récit de vie de l'humain Daniel 1, ressemblant à l'organisation invariante des systèmes vivants autopoiétiques, et des récits de vie des néo-humains, ressemblant à la structure variable de ces systèmes, mais ne relèvent distinctement d'aucun de ces deux espaces. Il ne s'agit pas de poches quantitatives qui seraient les productions finies, les marqueurs d'individualité du texte qui se dynamise, mais de formations, de fissures qui ne cessent de se nouer dans les mouvements de variation auto-créatifs à l'œuvre dans ce régime complexe d'enchevêtrement en train de naître.

Dans les cavités de ces poches se dévoilent des agencements volatils, à "potentialités oscillantes » (PI, 401) qui génèrent les mouvements auto-créatifs du système dont nous allons donner un exemple : l'installation artistique créée par Vincent, l'artiste de la secte élohimite. Celle-ci est créée dans un hangar sans fenêtres et incarne une salle d'intermédiation vers l'espace de la nouvelle humanité, tout en relevant de l'espace référentiel du récit de vie de Daniel 1 :

Dès que j'eus ouvert la porte hermétique, blindée, qui menait à l'intérieur, je fus ébloui par une lumière aveuglante, et pendant trente secondes je ne distinguai rien; (...) J'avais l'impression de me mouvoir à l'intérieur d'un espace laiteux, isotrope, qui se condensait parfois, subitement, en micro-formations grenues - en m'approchant je distinguais des montagnes, des vallées, des paysages entiers qui se complexifiaient rapidement puis disparaissaient presque aussitôt, et le décor replongeait dans une homogénéité floue, traversée de potentialités oscillantes. (...) Tournant mon regard vers la droite j'aperçus une silhouette qui répétait chacun de mes mouvements, qui ne se distinguait de la blancheur éblouissante de l'atmosphère que par un blanc légèrement plus mat. J'en ressentis une légère inquiétude : la silhouette disparut aussitôt. Mon inquiétude se dissipa : la silhouette 
se matérialisa à nouveau, comme surgie du néant. Peu à peu je m'habituai à sa présence, et continuai mon exploration ; il me paraissait de plus en plus évident que Vincent avait utilisé des structures fractales, je reconnaissais des tamis de Sierpinski, des ensembles de Mandelbrot et l'installation elle-même semblait évoluer à mesure que j'en prenais conscience. Au moment où j'avais l'impression que l'espace autour de moi se fragmentait en ensembles triadiques de Cantor la silhouette disparut, et le silence devint total. Je n'entendais même plus ma propre respiration, et je compris alors que j'étais devenu l'espace ; j'étais l'univers et j'étais l'existence phénoménale, les microstructures étincelantes qui apparaissaient, se figeaient, puis se dissolvaient dans l'espace faisaient partie de moi-même, et je sentais miennes, se produisant à l'intérieur de mon corps, chacune de leurs apparitions comme chacune de leurs cessations (PI, 400, 401, 402).

Cette installation artistique a un effet régulateur dans la création du régime complexe de La Possibilité, puisqu'elle configure la dynamique d'interaction des espaces alternatifs de la fiction, ce qui est dû aux potentiels métastables de ses «micro-formations grenues». Les fractales de Sierpinski, Mandelbrot et Cantor, ainsi que la poésie qui sous-tendent cette création illustrent ces potentiels à l'œuvre.

Les fractales sont pour Varela des ensembles aptes à saisir visuellement le fonctionnement étrange de la clôture opérationnelle des systèmes vivants autopoiétiques qui ne se limite pas à la stabilité d'un système dynamique, c'est-à-dire à l'homéostasie, mais tend continuellement à l'exploitation et au maintien de la métastabilité :

Dans une unité munie d'une clôture opérationnelle, un comportement cohérent et bien distinct présente, en fait, une nature particulière : d'un côté, il apparaît comme une opération de l'unité ; de l'autre côté, quand on tente d'examiner l'origine d'une telle opération, on ne trouve rien d'autre qu'une itération infinie de cette opération; elle ne commence nulle part et ne finit nulle part. (...) Avec l'exemple d'un fractal, on a tous les ingrédients, sous forme visuelle, pour voir comment la clôture d'un processus peut conduire : a) à une cohérence, toujours distribuée et jamais totalement présente mais compréhensible comme figure "mythique" ; b) à des propriétés émergentes au niveau de l'unité, qui ne résultent pas de la simple addition des propriétés des composants qui participent au processus (Varela, $1989 b$ : 25-26).

Les structures fractales de l'installation de Vincent traduisent donc le travail de variation autopoiétique qui ne cesse de configurer le monde émergeant de La Possibilité.

Par son potentiel de joindre «l'illimité » (Houellebecq, 1997 : 36), la poésie a également un rôle de participation active dans ce travail ${ }^{11}$ en opérant des ligatures impondérables, en imprégnant l'écriture de "son absurdité créatrice» (ibid.). La poésie, note Houellebecq, « brise la chaîne des causes et joue constamment avec la puissance explosive de l'absurde, mais elle n'est pas l'absurdité. Elle est l'absurdité rendue créatrice ; créatrice d'un sens autre, étrange mais immédiat, illimité, émotionnel » (ibid.).

Grâce à sa nature dynamique à l'œuvre, dépassant l'opposition entre organisation et structure $^{12}$, entre stabilité et dynamisme, la poche formée par l'agencement artistique de Vincent, avec ses structures fractales et ses échos poétiques, peut donc être conçue comme une posture de spécification de ce monde complexe naissant en tant que clôture opérationnelle, c'est-à-dire en tant que système, pensé à partir de l'ouverture, de la transformation métastable.

28 À travers l'exemple de l'œuvre de Vincent, nous avons pu montrer qu'une approche cognitive à travers le modèle de l'autopoï̀se nous permet de penser ce roman complexe, 
non pas comme une structure qui devient dynamique par rapport à une stabilité première, mais à partir du chaotique, du non-standard.

\section{Littérature et cognition : quelle légitimité ?}

La lecture du roman de Houellebecq à travers les sciences cognitives montre que l'apport d'un transfert de concepts du domaine cognitif au domaine littéraire se justifie par une légitimité méthodologique, qui consiste à réactualiser les méthodes de cartographie du texte en accordant plus d'importance à l'imprévisible, au mouvant, donc à une lecture dynamique. En ayant cette approche, la lecture devient un champ ouvert à la créativité, faisant appel aux concepts de la cognition qui modélisent l'autocréation, pour éclaircir les gestes de singularisation du texte littéraire. En ce sens, les concepts cognitifs ne servent pas à ériger une méthode systématique pour expliquer les romans, mais fonctionnent plutôt comme des outils qui permettent de penser le texte littéraire à partir d'un système auto-créatif en émergence.

Une telle approche de la littérature à travers le dispositif cognitif est en voie de légitimation depuis les années quatre-vingt, ce dont témoignent différents groupes de recherche (comme le CRLC, le Centre de recherche sur la littérature et la cognition de L'Université Paris 8, l'équipe de recherche Poétique cognitive de l'Université de Szeged, etc.) et de revues spécialisées (Théorie littérature enseignement (TLE), Substance, etc.).

Mais grâce à cette approche, la littérature enrichit également le champ de recherche de la cognition, puisqu'elle fonctionne comme un angle spécifique, à partir duquel il est possible de mieux découvrir le fonctionnement protéiforme et mouvant des mécanismes cognitifs. L'apport du fait littéraire au domaine de la cognition réside donc dans le fait qu'il permet une cartographie littéraire de la cognition, autrement dit, la compréhension des processus cognitifs à travers son propre cadre, celui de la narration. Ce cadre narratif peut même devenir une source qui permette l'avancement de nouvelles hypothèses sur le fonctionnement de la cognition, conduisant à un renouveau d'approches et de concepts dans le domaine des sciences cognitives.

Cette lecture transversale génère donc un circuit d'enrichissement mutuel entre la littérature et les sciences de la cognition, ce qui augmente leur potentiel de régénérescence à travers l'autre. Cependant, le premier volet de cette interférence, c'està-dire l'approche du champ littéraire à travers les modèles cognitifs a été plus largement explorée, d'où un renouvellement méthodologique en littérature (en français et étrangère) et une légitimité culturelle bien-fondée. Le deuxième volet, malgré sa portée de redistribution et de revitalisation des approches en sciences cognitives à travers les possibilités offertes par la narration, n'a pas encore été vraiment exploité. Il reste pour l'instant dans un état hypothétique, en quête de légitimité et de reconnaissance. 


\section{BIBLIOGRAPHIE}

ABRIOUX, Yves (1990). « Présentation », TLE Littérature et connaissance, n 8, pp. 5-9.

ATLAN, Henri, CASTORIADIS, Cornélius, DOMENACH, Jean-Marie et al. (1987). Création et désordre.

Paris : L'Originel.

Baudelaire, Charles (1999). « La mort des pauvres », in Claude Pichois (org). Les Fleurs du Mal. Paris : Gallimard.

[Dictionnaire des religions et des mouvements philosophiques associés] [on-line], [disponible le 02/02/2016] <URL : http://atheisme.free.fr/Religion/Definition_r.htm>.

HOUELLEBECQ, Michel (1998). «L'absurdité créatrice », in Interventions, Paris : Flammarion.

HouellebeCQ, Michel (2005). La Possibilité d'une île. Paris : Fayard.

KREMER-MARIETTI, Angèle (2012). « Réflexions sur l'autopoïèse » [Revue de philosophie et de sciences humaines] [on-line], DOGMA, [disponible le 02/02/2016] <URL : http://www.dogma.lu/ pdf/AKM-Autopoiese.pdf>.

VAn WeSemAel, Sabine (2005). Michel Houellebecq. Le Plaisir du texte. Paris : L'Harmattan.

VAN WESEMAel, Sabine, CLÉMENT, Murielle Lucie (2007). Michel Houellebecq sous la loupe. Amsterdam, New-York : Rodopi.

VARELA, Francisco, MATURANA, Humberto, URIBE, R. (1974). « Autopoiesis: The organization of living systems, its characterization and a model », Biosystems, vol. 5 / n 4, pp. 187-196.

VARELA, Francisco (1989a). Invitation aux sciences cognitives. Paris : Seuil.

VARELA, Francisco (1989b). Autonomie et connaissance, Essai sur le vivant. Paris : Seuil.

VARELA, Francisco, THOMPSON, Evan, ROSCH, Eleanor (1993). L'inscription corporelle de l'esprit. Paris : Seuil.

VARELA, Francisco (1999). « Quatre phares pour l'avenir des sciences cognitives », TLE Dynamique et cognition : nouvelles approches, $\mathrm{n}^{\circ} 17, \mathrm{pp} .7-23$.

VIARD, Bruno (2008). Houellebecq au laser : La faute à Mai 68. Nice : Ovadia.

WESTPHAL, Bertrand (2007). La Géocritique, Réel fiction espace. Paris : Minuit.

\section{NOTES}

1. Ce modèle apparaît pour la première fois dans un article de Varela, Maturana et Uribe en 1974 (cf. bibliographie).

2. De l'anglais to enact qui veut dire faire-émerger.

3. Il s'agit du cognitivisme qui postule que la cognition est un système de manipulation de symboles, à la manière des ordinateurs, et du connexionnisme qui considère la cognition comme l'émergence d'un état global basé sur la connexion permanente d'éléments locaux (les groupes de neurones) dans un réseau distribué (cf. Varela, 1989a).

4. Le mot d'ailleurs vient du grec auto (soi-même) et poièsis (production, création). 
5. La notion d'homéostasie vient du grec stasis (état, position) et homoios (égal, semblable à). Le concept d'homéostasie décrit la tendance d'un système à revenir au même état stable.

6. Dans le cadre de la cybernétique, le concept de métastabilité se réfère à l'état d'indétermination d'un système qui a pour but d'atteindre l'homéostasie. Or, dans le cas du modèle autopoiétique, la métastabilité ne renvoie pas à une tendance au maintien du même état, mais à un processus de reconfiguration continuelle à références internes, cultivant des agencements imprévisibles, dynamiques.

7. Ce roman a été récompensé par le prix Interallié en 2005. Par la suite, les références à ce roman seront notées $P$.

8. Il s'agit d'une secte, inspirée de celle des räeliens. Le mouvement raëlien, fondé par Claude Vorilhon en 1946, a été catégorisé comme secte dans le rapport parlementaire français de 1996. Leur fondateur prétend avoir rencontré des extraterrestres, les Élohim, qui lui ont confié la mission de répandre leur enseignement parmi les fidèles dans le monde entier. Vorilhon devient donc le gourou de la secte raëlienne (son nouveau prénom Raël signifie «messager ») et prêche que toute forme de vie sur Terre est due aux Élohim grâce à leur maîtrise avancée du génie génétique (cf.http://atheisme.free.fr/Religion/Definition_r.htm).

9. Westphal distingue le seuil (limen) de la frontière (limes) : « le seuil suppose le franchissement libre, contrairement à la frontière, qui peut se révéler étanche » (Westphal, op. cit., p. 163).

10. «L'explication opérationnelle » des systèmes vivants (c'est-à-dire par clôture opérationnelle) s'oppose à « l'explication symbolique » (les systèmes à entrées-sorties) qui est aussi utilisée.

11. Pour créer son œuvre, Vincent s'inspire effectivement de La mort des pauvres de Baudelaire ( Les Fleurs du Mal) : C'est la mort qui console, hélas ! et qui fait vivre; / C'est le but de la vie, et c'est le seul espoir / Qui, comme un élixir, nous monte et nous enivre, / Et nous donne le cœur de marcher jusqu'au soir ; / À travers la tempête, et la neige, et le givre, / C'est la clarté vibrante à notre horizon noir ; / C'est l'auberge fameuse inscrite sur le livre, / Où l'on pourra manger, et dormir et s'asseoir... (PI, 400).

12. Nous nous référons à l'organisation et à la structure des systèmes autopoiétiques.

\section{RÉSUMÉS}

Cet article rend compte de l'apport et de la légitimité d'une lecture transversale entre la littérature et les sciences cognitives, à travers un roman français contemporain, La Possibilité d'une île de Michel Houellebecq. En s'appuyant sur le modèle de l'autopoï̀se, développé par Francisco Varela et Humberto Maturana, nous proposons de lire ce texte à partir d'un système chaotique, métastable. Enfin, nous soulignons la double portée de cette interférence entre la littérature et la cognition: d'un côté un renouvellement méthodologique en cartographie des textes grâce au dispositif cognitif, de l'autre, la revitalisation des approches et des concepts en sciences de la cognition à travers le cadre narratif.

This study reports on the contribution and the legitimacy of a transversal reading between literature and cognitive science, through a contemporary french novel, La Possibilité d'une île (The Possibility of an Island) by Michel Houellebecq. Relying on the autopoietic model, developed by Francisco Varela and Humberto Maturana, we propose to read this text from a chaotic, metastable point of view. Finally, we underline the double significance of this interference between literature and cognition: on one hand, a methodological renewal in literary cartography 
thanks to cognitive models, on the other hand, the revitalization of the approaches and concepts in cognitive science through a narrative context.

INDEX

Mots-clés : littérature, cognition, autopoï̀se, métastable, légitimité, méthodologie

Keywords : literature, cognition, autopoiesis, metastable, legitimacy, methodology

\section{AUTEUR}

\section{GABRIELLA BANDURA}

Un. de Szeged/Un. Paris 8

gbandura17[at]@yahoo.fr 